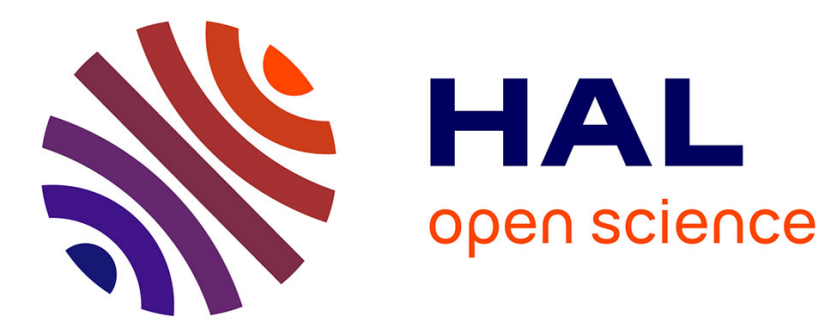

\title{
Time-resolved tomographic measurements of temperatures in a thermal plasma jet \\ J Hlína, J Šonský
}

\section{To cite this version:}

J Hlína, J Šonský. Time-resolved tomographic measurements of temperatures in a thermal plasma jet. Journal of Physics D: Applied Physics, 2010, 43 (5), pp.55202. 10.1088/0022-3727/43/5/055202 . hal-00569752

\section{HAL Id: hal-00569752 \\ https://hal.science/hal-00569752}

Submitted on 25 Feb 2011

HAL is a multi-disciplinary open access archive for the deposit and dissemination of scientific research documents, whether they are published or not. The documents may come from teaching and research institutions in France or abroad, or from public or private research centers.
L'archive ouverte pluridisciplinaire HAL, est destinée au dépôt et à la diffusion de documents scientifiques de niveau recherche, publiés ou non, émanant des établissements d'enseignement et de recherche français ou étrangers, des laboratoires publics ou privés. 


\title{
Time-resolved tomographic measurements of temperatures in a thermal plasma jet
}

\author{
J Hlína and J Šonský \\ Institute of Thermomechanics AS CR, v.v.i., Academy of Sciences of the Czech Republic, \\ Dolejškova 5, 18200 Praha 8, Czech Republic
}

\section{e-mail: hlina@it.cas.cz}

PACS numbers: 52.70.Kz, 52.35.Ra, 52.75.Hn

Keywords: Thermal plasma jet, optical diagnostics, temperature distribution.

\begin{abstract}
Temperatures in a thermal plasma jet were measured using an experimental set-up providing time-resolved scans of plasma optical radiation near $650 \mathrm{~nm}$ from 4 directions. The acquired data were used for reconstructions of time-resolved temperature distributions in measurement planes perpendicular to the plasma jet axis. The plasma torch was fed by the d.c. power source with a substantial ripple voltage modulation which leads to significant differences between the temperature distributions according to the actual phase of the arc current.
\end{abstract}




\section{Introduction}

Thermal plasma generated by d.c. plasma torches has become an important tool in many technologies, such as plasma spraying, chemical vapour deposition, waste destruction, synthesis of nanoparticles etc. Progress in these technologies is, among other things, closely connected with the development of new approaches aiming at describing realistic conditions in thermal plasmas [1]. One of the most important parameters needed for the plasma description is temperature. The possibility of representation of thermal plasma by a single temperature value depends on the validity of LTE (local thermal equilibrium) assumption, which is doubtful in many cases [2]. The achievement of LTE is difficult especially when plasma includes more components or when the cold gas is injected. The assumption about LTE is also not generally valid on the boundaries of the plasma jet where the flow is influenced by the entrainment of the cold gas from the ambient atmosphere [3]. Nevertheless, in some conditions the thermal plasma jets may be still investigated using the LTE assumption [4]. Besides the problem with LTE, thermal plasma jets are characterized by some more properties complicating both the theoretical and experimental investigations. Arc attachment instabilities and turbulence on the jet boundaries result in time-dependent plasma characteristics and disturbances of the circular symmetry. This leads to the necessity of 3D modeling taking into account various sources of instabilities and development of new experimental tomographic methods with high temporal resolution.

Recent theoretical models are able to simulate three-dimensional and time-dependent flow inside a d.c. arc plasma torch [5]. In the models the anode attachment process plays an important role as a source of plasma jet instabilities. In other types of torches the instability may also develop due to the arc root movement inside a hollow cathode [6]. Such models may even include a two-temperature thermal non-equilibrium [7]. In spite of these 3D, non-stationary approaches some recent theoretical studies assume stationary conditions and axisymmetric plasma flow and the physical description concentrates just on the influence of non-equilibrium conditions (two-temperature models $[8,9]$ ). Some models include also the simulations of the injection of particles and carrier gas into the plasma jet [10].

Calculation of plasma temperature is one of principal goals of theoretical models and finding of its credible values is also an important challenge for experimental investigations of thermal plasma jets. Real conditions in thermal plasma jets including possible LTE disturbance, non-stationarity and asymmetry pose very difficult claims on experimental set-up and evaluation methods. Experimental investigation methods are not so far able to respect all the above mentioned conditions together and it is thus necessary to consider the results of temperature measurements with relevant care. The most of methods are based on optical diagnostics because of significant limitations connected with intrusive methods (Langmuir and enthalpy probes). Optical emission spectroscopy (OES) belongs to traditional methods of plasma temperature measurements and usually makes use of measurement on several spectral lines and temperature is then derived e.g. from the Boltzmann plot [11]. The application of this method to temperature measurements in the non-stationary plasma was realized by a rotating mirror so that it was possible to measure simultaneously the intensity of several spectral lines with a spectrometer [12]. A spatially resolved method using absolute intensity measurements on one spectral line through an interference filter and using for the detection a photodiode array was described in [13]. A significant advance in the measurements of spatial distribution of temperatures in the plasma jet has been achieved lately thanks to the application of CCD cameras. A high-speed CCD camera equipped with a narrow-band filter was used for scanning temperature fields in arc plasma [14, 15]. Singledirectional observations of the jet applied in these works assume cylindrical symmetry of the jet. It was shown that in some conditions the temperature field in a plasma jet can be simply mapped by a colour CCD camera comparing the intensities of different colour image components [16]. This idea was confirmed by theoretical evaluations of plasma emissivity in large spectral bands [17]. Thomson scattering experiments are also an important source of information about arc plasma temperatures, e.g. when studying various anode attachment modes [18] or LTE conditions in the anode region [19].

The entrainment of the surrounding atmosphere into the jet [20] influences significantly the possibilities of temperature measurement especially at the jet boundaries and tail. The entrainment may be diagnosed by Thomson scattering [21], by spectrally resolved measurements detecting the radiation of the core and surrounding gases [22] or by enthalpy probes [23]. 
It seems that at present the achievements in theoretical works which are able to predict 3D, non-stationary and non-equilibrium characteristics of thermal plasma jets are not fully coped with the capability of experimental investigations which still do not afford complex 3D and time- resolved plasma characteristics respecting possible asymmetry of the jet. As far as some recent papers have laid foundations of the tomographic diagnostics of asymmetrical plasma radiation sources [24, 25], it is now possible to apply these methods also for the development of new methods for measurements of plasma temperatures respecting complex 3D and non-stationary character of the plasma flow. The aim of our work is to contribute to some progress in this field. For this purpose we have adapted a system for multi-directional observations of plasma jets [26] which is able to provide distributions of plasma radiation in planes perpendicular to the plasma jet axis with a high temporal resolution. The system was equipped with interference filters in order to detect plasma radiation in a specific spectral band. In this paper we show possibility to use such experimental system and method based on evaluations of absolute intensities of plasma radiation in a limited spectral interval for measurements of timeresolved temperature distributions in an argon plasma jet showing various types of instabilities.

\section{Experimental arrangement}

The argon plasma jet was blown out in air by a vertically oriented plasma torch (figure 1) working with the arc length $7 \mathrm{~mm}$, nozzle diameter $6 \mathrm{~mm}$ and d.c. arc current 200 A. Argon was injected in the torch axially and tangentially with steady axial flow rate $10 \mathrm{slm}$ and variable tangential flow rates in the range 17-34 slm. The plasma jet radiation was projected through 4 objective lens and interference filters with the maximal transmittance at $650 \mathrm{~nm}$ and spectral width approx. $80 \mathrm{~nm}$ on face areas of linear arrays of optical fibers and photodiodes arranged at 4 directions and 2 levels. The optical arrays (each consisting of 16 elements) detected the plasma radiation at levels 2 and $4 \mathrm{~mm}$ above the nozzle. This experimental set-up provided records of plasma jet optical radiation with the sample rate $468 \mathrm{kHz} / \mathrm{channel}$ and record lengths $1 \mathrm{Msample} / \mathrm{channel}$. The transformation of the measured radiation intensities to the absolute values was carried out via the calibration using a tungsten ribbon lamp.

\section{Method of temperature evaluation}

The distributions of plasma radiation in the measurement planes were reconstructed using a method of interpolation of the measured data and the inverse Radon transform procedure in MATLAB environment described more in detail in [25]. MATLAB procedure uses the filtered back-projection algorithm and assumes the input data in the form of arrays representing the object projections taken at a set of angles with equal increments. The optimal behaviour of the procedure required some preliminary processing of the input data. The experimental data acquired in each of scanning directions were interpolated in two steps; first by splines adding additional points between the measured ones in order to suppress oscillations of high-order polynomials used then in the second step for the interpolation of the whole side-on profiles. For the interpolation of the data presented in this work we used polynomials of the $24^{\text {th }}$ degree. Four interpolated profiles representing the projections of the plasma jet radiation in 4 measuring directions were then amended by 4 additional intermediate (fictive) profiles represented by polynomials whose coefficients were acquired by interpolation of polynomial coefficients found for the four original side-on profiles. It was found that such procedure enhancing the number of input profiles entering into the succeeding inverse Radon transform leads to resulting planar distributions which are more authentic especially in boundary regions. The data arrays needed as an input for the inverse Radon procedure were then deducted as polynomial values in 50 equidistant points and the reconstructed radiation distributions had the form of matrices with the default size of $35 \times 35$ elements. The values of radiation intensities in these elements were adjusted by a normalization procedure ensuring that the integral radiation of the cross section (represented by the sum of the matrix) was equal to the integral radiation measured in a single direction. Tests on models have shown the ability of this method to reconstruct rather complicated structures with more than one maximums [26]. Therefore we assume a correct reconstruction of more simple, single-maximum distributions representing the radiation in the plasma jet cross-sections. 
Plasma temperatures were found by the comparison of the measured plasma radiation values with the results of theoretical calculations of argon plasma radiation in the spectral range transmitted by interference filters (example of transmittance curve is in figure 2) as a function of temperature in the range $8000-12000 \mathrm{~K}$. The calculations assume optically thin thermal plasma. Good conditions for low absorption of radiation in our spectral range are based on occurrence of relatively weak spectral lines and they are supported by short optical paths $(<5 \mathrm{~mm})$ where temperatures exceed $9000 \mathrm{~K}$. For such temperatures the radiation escape factors show a significant drop [17]. Local thermal equilibrium (LTE) may be assumed in our conditions, correspondingly to similar plasma torches [4], in the plasma jet central part near the torch exit. The influence of possible LTE violations at the plasma jet boundaries on temperature evaluation is discussed hereinafter in Chapter 5.

The radiation in the considered spectral range 580-730 nm includes 115 Ar I lines. The plasma optical line emission $\mathcal{E}_{l}$ transmitted by the interference filter was computed (in $\mathrm{Wm}^{-3} \mathrm{sr}^{-1}$ ) as

$$
\varepsilon_{i}=\sum_{i=1}^{N} t(i) \frac{h v(i)}{4 \pi} \frac{g_{k}(i)}{\sigma} A_{k}(i) n_{0} \exp \left(-\frac{E_{k}(i)}{k T}\right),
$$

where $\mathrm{N}$ is the number of lines, $t(i)$ transmittance of interference filter at the wavelength of $\mathrm{i}^{\text {th }}$ spectral line, $h$ Planck constant, $v(i)$ wavenumber, $g_{k}(i)$ statistical weight of upper level, $\sigma$ partition function (we have taken $\sigma=1$ ), $A_{k}(i)$ Einstein coefficient for spontaneous deexcitation of the upper level, $n_{0}$ concentration of neutral atoms, $E_{k}(i)$ energy of the upper level, $k$ Boltzman constant and $T$ temperature. The values of $v(i), g_{k}, A_{k}(i), E_{k}(i)$ for the considered lines were taken from NIST tables [27]. The lines in the range of maximal filter transmittance $(610-690 \mathrm{~nm})$ are relatively weak with insignificant selfabsorption and the region is thus suitable for the applied measurement method (see [17]).

The calculations of the plasma radiation included continuum emission $\varepsilon_{c}$ which was computed (in $\mathrm{Wm}^{-3} \mathrm{sr}^{-1}$ ) using the formula

$$
\varepsilon_{c}=\xi \frac{C}{\lambda^{2}} \frac{n_{e}^{2}}{\sqrt{T}} t_{f}
$$

where $\xi$ is the Biberman factor, $C$ a constant, $\lambda$ wavelength, $n_{e}$ electron concentration, $T$ temperature and $t_{f}$ is integrated filter transmittance. According to Dgheim [28] we have taken $C=1.63 \times 10^{-43}$ $\mathrm{Wm}^{4} \mathrm{~K}^{0.5} \mathrm{sr}^{-1}$ and $\xi=2$. The neutral and electron concentrations were calculated from the ideal gas and Saha equations. The total plasma radiation transmitted through the interference filter $\varepsilon_{=}=\varepsilon_{l}+\mathcal{E}_{c}$ was then calculated as a function of temperature (figure 3).

The recorded side-on plasma radiation intensities were converted to absolute intensities with the aid of a tungsten ribbon calibration lamp. The resulting planar intensity distributions and theoretical dependence of radiation on temperature were then used to acquire plasma temperatures.

\section{Results}

The experimental data may capture the dynamical behaviour of the plasma jet in planes perpendicular to the jet axis in a very long time period (approx. $2 \mathrm{~s}$ ) compared to the basic periods characteristic for the plasma jet dynamics which is an important condition for the possibility to study the underlying processes. The most significant instabilities of the plasma jet are caused by ripple modulation of the arc current coming from the three-phase rectifier used as a power source and turbulent phenomena occurring on the plasma jet boundaries [29]. In our plasma torch these two types of instabilities are combined with acoustic oscillations originating in the arc chamber [30]. Frequency spectra obtained by the fast Fourier transform of reconstructed optical radiation distributions (analyzed from $2^{14}=16384$ distributions, corresponding time interval $35 \mathrm{~ms}$ ) at the axis and at the plasma edge ( 3 $\mathrm{mm}$ from the axis) at the tangential flow rate $23.5 \mathrm{slm}$ and distance $4 \mathrm{~mm}$ from nozzle are in figure 4 . The acoustic (Helmholtz type) oscillations with the basic frequency approx. $3.5 \mathrm{kHz}$ have maximal amplitudes at the jet axis. The second harmonic of this frequency at approx. $7 \mathrm{kHz}$ coincides with characteristic frequency of the shear layer instability and shows thus maximal amplitudes in the 
boundary regions. The spectra do not indicate in our case the presence of any significant oscillations with frequencies higher than $25 \mathrm{kHz}$.

Slightly different peak current in each of the three phases leads to arc current and plasma radiation modulation showing the basic recurrence period $10 \mathrm{~ms}$ (corresponding to $100 \mathrm{~Hz}$ ) composed of three periods $3.33 \mathrm{~ms}$ long (corresponding to the frequency $300 \mathrm{~Hz}$ ). Arc current ripple (ratio of a.c. amplitude to d.c. component) was approx. $40 \%$. The turbulence on plasma jet boundaries is characterized by much higher frequencies up to several tens of $\mathrm{kHz}$. The power source modulation is decisive for the modulation of maximal plasma temperature in the measurement cross section. Figure 5 and figure 6 show $10 \mathrm{~ms}$ long evolution of maximal temperatures $2 \mathrm{~mm}$ from the nozzle for tangential gas flow rates 17 and $23.5 \mathrm{slm}$, respectively. Whereas the temperature modulation depth at $17 \mathrm{slm}$ is about $800 \mathrm{~K}$ (temperature range approx. $9600-10400 \mathrm{~K}$ ), the increase of the flow rate to $23.5 \mathrm{slm}$ reduces the modulation depth to about $600 \mathrm{~K}$ (temperature range approx. $10050-10650 \mathrm{~K}$ ). The locations of points with maximal temperatures (maximums of instantaneous temperature planar distributions) are not always identical with the location of the plasma torch axis because of strong disturbances influencing the temperature distributions especially in the phases of low arc current. Short sequences comprising 8 successive temperature distributions (intervals $2.14 \mu \mathrm{s}$ ) showing the evolution in the high and low current phases of the arc current for the tangential flow rate $17 \mathrm{slm}$ are in figures 7 and 8. The comparison of temperature distributions in the high current (figure 7) and low current (figure 8) phases shows significant differences. While the distributions in the high current phase are relatively stable and symmetrical, the distributions in the low current phase are characterized by complicated asymmetrical shapes and fast changes. Average temperature distributions show relatively good circular symmetry. This is demonstrated in figure 9 showing an average temperature distribution for $10 \mathrm{~ms}$ long time interval including instantaneous distributions from figures 6 and 7 . The temperature fluctuations evaluated as standard deviations (std) are highest at the boundaries where the standard deviation of temperature can be as high as $400 \mathrm{~K}$. An example showing a map of temperature standard deviations $4 \mathrm{~mm}$ from the nozzle for tangential flow rate $17 \mathrm{slm}$ is in figure 10 . Boundary regions with highest fluctuations are characterized by mean temperatures about $8600-8700$ $\mathrm{K}$. The evaluation of temperature distributions in the basic recurrent time period $10 \mathrm{~ms}$ may yield data characterizing both the mean temperature values and their fluctuations in various parts of the plasma jet. Examples of such results are shown in Table 1. The table contains the comparison of plasma jet characteristics at tangential flow rates 17 and $23.5 \mathrm{slm}$ at distances 2 and $4 \mathrm{~mm}$ from the plasma torch nozzle. The characteristics include the mean of temperature maximums, standard deviation of these maximums (fluctuations of maximal temperature), dimension of areas where the mean temperatures exceed $8600 \mathrm{~K}$, total dimension of areas characterized by very high temperature fluctuations (standard deviation of temperatures higher than $300 \mathrm{~K}$ ) and mean temperature of these areas.

Table 1. Mean temperature values and their fluctuations in various parts of the plasma jet.

\begin{tabular}{|c|c|c|c|c|}
\hline & \multicolumn{2}{|c|}{ tangential flow rate $17 \mathrm{slm}$} & \multicolumn{2}{|c|}{ tangential flow rate $23.5 \mathrm{slm}$} \\
\hline & distance $2 \mathrm{~mm}$ & distance $4 \mathrm{~mm}$ & distance $2 \mathrm{~mm}$ & distance $4 \mathrm{~mm}$ \\
\hline mean temperature at maximum $[\mathrm{K}]$ & 10073 & 10013 & 10384 & 10387 \\
\hline std of temperature at maximum $[\mathrm{K}]$ & 243 & 231 & 193 & 185 \\
\hline $\begin{array}{l}\text { dimension of area with mean } \\
\text { temperature }>8600 \mathrm{~K}\left[\mathrm{~mm}^{2}\right]\end{array}$ & 26 & 32 & 30 & 35 \\
\hline $\begin{array}{l}\text { dimension of areas with std } \\
\text { of temp. }>300 \mathrm{~K}\left[\mathrm{~mm}^{2}\right]\end{array}$ & 9 & 3 & 8 & 3 \\
\hline $\begin{array}{l}\text { mean temperature in the area of } \\
\text { temperature std }>300 \mathrm{~K}[\mathrm{~K}]\end{array}$ & 8714 & 8697 & 8622 & 8648 \\
\hline
\end{tabular}

Differences between plasma jet characteristics depicted in Table 1 for the distances 2 and 4 $\mathrm{mm}$ from the nozzle are small except rather rapid axial decrease of central temperature at a lower gas flow rate. Higher maximal temperatures at the flow rate $23.5 \mathrm{slm}$ compared to those 
at $17 \mathrm{slm}$ are due to cooling of the plasma in the anode channel (length $22 \mathrm{~mm}$ ) which causes more significant decrease of temperature at lower gas velocities. In addition to that, maintenance of constant arc current during our experiments results in an increase of input power for a higher gas flow rate because of higher mean arc voltage $(30 \mathrm{~V}$ at $17 \mathrm{slm}, 33 \mathrm{~V}$ at $23.5 \mathrm{slm})$. Evaluations of temperature fluctuations have shown that a lower gas flow rate leads to higher fluctuations of the maximal temperature and penetration of most intense fluctuations (characterized by std $>300 \mathrm{~K}$ ) into regions closer to the jet axis.

\section{Discussion}

The reliability of results acquired by the method of tomographic temperature evaluation based on measurements of absolute radiation intensities depends, similarly as in the symmetric case when Abel inversion is applied, on the credibility of atom concentration values used in the computations. In our case the concentration of argon atoms in the plasma jet boundary region is influenced by mixing of argon and air. Argon concentration is thus reduced mainly in favour of nitrogen and oxygen. These species may affect our results either by their own radiation or, assuming constant pressure, by reducing the concentration of argon.

The temperatures in the mixing region are characterized by values below $9000 \mathrm{~K}$ which are not sufficient for a significant degree of nitrogen and oxygen dissociation (dissociation energies for $\mathrm{O}_{2}$ $-5.1 \mathrm{eV}, \mathrm{N}_{2}-9.76 \mathrm{eV}$ ). The main part of the optical radiation produced by these gases in the boundary region is thus radiated in the form of several molecular rotational-vibrational bands. Our previous measurements [22] have shown that the most significant band is formed by the second positive system of nitrogen molecules with wavelengths near $290 \mathrm{~nm}$. We have found no atomic lines of nitrogen or oxygen. The effect of nitrogen and oxygen radiation in the spectral band transmitted by our interference filters centered at $650 \mathrm{~nm}$ is thus negligible.

The decrease of argon concentration in the mixing region leads to lower measured radiation than is predicted by formulas (1) and (2) for pure argon. The influence of air (if its amount is known) can be corrected either by a proper change of concentrations in the formulas or (due to the linear dependence of radiation on concentrations) by a corresponding correction of measured radiation intensities. Previous theoretical and experimental investigations of a similar plasma torch $[22,31]$ have shown insignificant air entrainment for plasma jet regions with distances less than $2 \mathrm{~mm}$ from the axis and $6 \mathrm{~mm}$ from the nozzle orifice. We have analyzed on one of measured radiation distributions ( $4 \mathrm{~mm}$ from the nozzle, argon tangential flow rate $23.5 \mathrm{slm}$ ) possible influence of air presence in the boundary region by calculations modeling entrainment of $0-50 \%$ air into the jet boundary region. The region was defined as a zone with radiation intensities $\mathrm{E}<\mathrm{E}_{0} / 3$, where $\mathrm{E}_{0}$ is the maximal intensity at the jet axis. This corresponds to regions with the distances from the axis higher than approx. 2 $\mathrm{mm}$. Due to the integral character of the Radon transform the correction of radiation intensities modeling air entrainment at the jet edge results in the decrease of transformed radiation distribution values in the center. As a result, the correction model assuming entrainment of some air in the plasma jet boundaries yields temperature values which are lower in the central region and higher at the boundary compared to the results assuming no entrainment. Table 2 shows results (maximal temperature $\mathrm{T}_{0}(\mathrm{en})$ at the axis, off-axis temperature $\mathrm{T}_{\mathrm{x}}(\mathrm{en})$ at a point characterized by radiation $\mathrm{E}=\mathrm{E}_{0} / 10$ - approx. $3 \mathrm{~mm}$ from the axis for entrainments defined as en $=0,10,20,50 \%$ of air in the boundary region and relative errors $\left.\Delta \mathrm{T}_{0}=\left(\mathrm{T}_{0}(\mathrm{en})-\mathrm{T}_{0}(\mathrm{en}=0)\right) / \mathrm{T}_{0}(\mathrm{en}=0), \Delta \mathrm{Tx}=\left(\mathrm{T}_{\mathrm{x}}(\mathrm{en})-\mathrm{T}_{\mathrm{x}}(\mathrm{en}=0)\right) / \mathrm{Tx}(\mathrm{en}=0)\right)$ yielded by these model calculations. The calculations indicate rather small effect of air entrainment on temperature values in the plasma jet central part. 
Table 2. Dependence of axial and edge temperatures

on relative concentration of air in the boundary region.

\begin{tabular}{|l|l|l|l|l|}
\hline$\%$ of air & $\mathrm{T}_{0}[\mathrm{~K}]$ & $\Delta \mathrm{T}_{0}[\%]$ & $\mathrm{T}_{\mathrm{x}}[\mathrm{K}]$ & $\Delta \mathrm{T}_{\mathrm{x}}[\%]$ \\
\hline 0 & 10610 & 0 & 8955 & 0 \\
\hline 10 & 10594 & -0.2 & 9010 & +1.2 \\
\hline 20 & 10579 & -0.3 & 9150 & +2.2 \\
\hline 50 & 10537 & -0.7 & 9331 & +4.2 \\
\hline
\end{tabular}

High temperature gradients in the boundary layer and mixing with the ambient atmosphere also result, according to many authors, in violations of LTE consisting in lower values of the gas temperature compared to the electron temperature determining the measured excitation temperature of argon atoms. This leads, contrary to the previous effect (quantified in Table 2), to the overestimation of the gas temperature in these regions and possible reduction of errors shown in Table 2. The main portion of continuum emission is due to the radiative recombination and the effect of the thermal disequilibrium on this part of calculation results is not significant.

Another possible source of the measurement error is the inequality of interference filters used for scans of radiation in individual measurement directions. Slightly different spectral characteristics of the filters (which were measured) result in shifted courses of theoretical temperature/radiation dependence used for the evaluation of measured radiation intensities. The maximal difference between the light transmitted by a single filter and the mean transmittance used for the calculations was $8.1 \%$ which leads to the error in temperature determination $1 \%$ at $10000 \mathrm{~K}$.

\section{Conclusion}

Tomographic methods with a high temporal resolution offer new possibilities in optical diagnostics of asymmetric and non-stationary thermal plasmas. Temperature measurements present a special problem of the diagnostic of such plasmas and they need careful consideration regarding the concrete gas properties and the choice of a proper spectral range suitable for measurements. The approach based on measuring plasma optical emission in large spectral intervals is necessary to ensure enough signal for the fast optical recording. In the case of argon plasma, we have chosen for the temperature measurements $80 \mathrm{~nm}$ wide spectral interval around $650 \mathrm{~nm}$ (characterized by almost balanced portions of continuum and line radiation) because of relatively weak spectral Ar I lines showing low self-absorption. The selection of the interval in the red part of the optical spectra is also advantageous due to relatively high sensitivity of our sensors in this range.

The application of the measurement method in the case of a thermal plasma jet fed from the d.c. power source with a significant voltage modulation has shown that it can bring new findings concerning short-time and spatial characteristics of temperature fields in a such plasma jet. While the cross-sectional distributions of plasma radiation (and thus also temperature) in the high-current phase (scanned at times near the arc current maximums) show almost circular symmetry, the distributions in the low-current phase are characterized by very complicated shapes and fast changes. These lowcurrent phase instabilities affecting significantly temperature fluctuations near the plasma jet axis are not however much pronounced in the 2D map of temperature standard deviations covering the basic recurrence period which is $10 \mathrm{~ms}$ long. Evaluations of temperature fluctuations in this or longer periods show maximal standard deviations on the plasma jet boundaries where the effect of turbulence is permanent throughout the considered time period. This illustrates the importance of spatially and time resolved measurements for the trustful diagnostics of thermal plasma jets respecting their dynamics. 
Regarding the credibility of temperature values in a thermal plasma jet acquired by measurements of plasma radiation in large spectral intervals, it is important to have in mind possible mixing with ambient atmosphere on the plasma jet boundaries and an increasing influence of the surrounding gas entrainment in regions farther from the nozzle. Due to this effect the resulting temperature distributions near the plasma jet core and not far from the nozzle may be considered as more reliable.

\section{Acknowledgement}

The work was supported by Institutional Research Plan AVOZ 20570509. The authors thank their colleague J. Šlechta for helpful discussions.

\section{References}

[1] Heberlein J 2002 New approaches in thermal plasma technology Pure Appl. Chem. 74 327-335

[2] Fauchais P, Vardelle A 2000 Pending problems in thermal plasmas and actual development Plasma Phys. Control. Fusion 42 B365-B383

[3] Russ S, Pfender E, Strykowski P J 1994 Unsteadiness and Mixing in Thermal Plasma Jets Plasma Chemistry and Plasma Processing 14 425-436

[4] Tu X, Chéron B G, Yan J H, Cen K F 2007 Electrical and spectroscopic diagnostic of an atmospheric double arc argon plasma jet, Plasma Sources Sci. Technol. 16 803-812

[5] Chazellas C, Moreau E, Mariaux G, Vardelle A 2006 Numerical modeling of arc behaviour in a dc plasma torch, High Temp. Mater. Proc. 10 393-405

[6] Freton P, Gonzales J J, Gleizes A, Conte D 2007 3D modelling of the cathodic arc root movement in a hollow cathode of a thermal plasma torch Proc. $28^{\text {th }}$ ICPIG (Prague) pp. 1770-1773

[7] Trelles J P, Heberlein J, Pfender E 2007 Non-equilibrium modelling of arc plasma torches J. Phys. D: Appl. Phys. 40, 5937-5952

[8] Kaminska A, Lopez B, Izrar B, Dudeck M 2008 Modelling of an argon plasma jet generated by a dc arc Plasma Sources Sci. \& Technol. 17035018

[9] Rat V, Murphy A B, Aubreton J, Elchinger M F, Fauchais P 2008 Treatment of non-equilibrium phenomena in thermal plasma flows J. Phys. D: Appl. Phys. 41183001

[10] He-Ping, Pfender E 2007 Three Dimensional Modeling of the Plasma Spray Process Jour. Thermal Plasma Spray Technol. 16(2) 245-260

[11] Sember V 2003 Spectroscopic study of a thermal plasma jet generated by a hybrid water-argon stabilized DC arc torch High Temp. Mater. Proc. 7 17-22

[12] Wen Hua Zhao, Jian Quan Li, Jiu Dun Yan 1997 Temperature Measurement of Nonsteady Arcs IEEE Trans. Plas. Sci. 25 828-832

[13] Hlína J, Šlechta J 1998 Temperature measurements in an oscillating plasma jet Proc. 11th Symposium on Elementary Processes and Chemical Reactions in Low Temperature Plasma (Low Tatras) vol 1 pp. 79-82

[14] Xu C M, Xao H M, Wu L 2005 Application of monochromatic image method in arc plasma diagnostic IEEE Trans. Plas. Sci. 33, 304-305

[15] Ma S L, Gao H M, Wu L, Zheng S M 2008 Time and spatially resolved spectroscopic measurement of temperatures in free-burning arc by monochromatic imaging Meas. Sci. Technol. 19 105602

[16] Hlina J, Cressault Y, Gleizes A, Gonzalez J J, Razafinimanana M 2004 Application of a Colour CCD Camera to Evaluations of Temperature Distribution in a Thermal Plasma Jet Proc. XV International Conference on Gas Discharges and their Applications (Toulouse) pp. 881-884 [17] Rouffet M E, Cressault Y, Gleizes A, Hlína J 2008 Thermal plasma diagnostic methods based on the analysis of large spectral regions of plasma radiation J. Phys. D: Appl. Phys. 41125204

[18] Yang G, Heberlein J 2007 Anode attachment modes and their formation in a high intensity argon arc Plasma Sources Sci. Technol. 16 529-542

[19] Zhu X, Redwitz M, Kieft E R, van der Sande M J, van der Mullen J J A M 2008 Thomson scattering measurements on an atmospheric Ar dc discharge lamp J. Phys. D: Appl. Phys. 37 736-743 [20] Russ P, Strykowski P J, Pfender E 1994 Mixing in plasma and low density jets Experiments in Fluids 16 297-307 
[21] Fincke J R, Crawford D M, Snyder S C, Swank W D, Haggard D C, Williamson R L 2003 Entrainment in high-velocity, high-temperature plasma jets. Part I: experimental results Int. Jour. Heat Mass Transf. 46 4201-4213

[22] Hlína J, Šonský J, Šlechta J 2007 Application of CCD cameras in investigations of mixing on boundaries of a thermal plasma jet Plas. Sci. Technol. 9 743-746

[23] Choi S, Hwang T H, Seo J H, Kim D U, Hong S H 2004 Effects of anode nozzle geometry on ambient air entrainment into thermal plasma jets generated by nontransferred plasma torch IEEE Trans. Plas. Sci. 32, 473-478

[24] Franceries X., Freton P., Gonzales J. J., Lago F., Masquère M 2005 Tomographic reconstruction of 3D thermal plasma systems: a feasibility study, J. Phys. D: Appl. Phys. 38, 3870-3884

[25] Hlína J, Chvála F 2006 On application of inverse Radon transform for diagnostics of asymmetric plasmatic radiation sources Acta Technica CSAV 51, 317-325

[26] Hlína J, Chvála F, Šonský J, Gruber J 2008 Multi-directional optical diagnostics of thermal plasma jets Meas. Sci. Technol. 19, 01540

[27] Ralchenko Yu, Kramida A E, Reader J and NIST ASD Team (2008). NIST Atomic Spectra Database (version 3.1.5). Available: http://physics.nist.gov/asd3. National Institute of St:

Technology, Gaithersburg, MD.

[28] Dgheim J 2007 Argon plasma jet continuum emission investigation by using different spectroscopic methods Plasma Sources Sci. Technol. 16 211-216.

[29] Gruber J, Hlína J, Šonský J, Chvála F 2007 FFT analysis of planar distributions of oscillations in a thermal plasma jet, International Conference on Phenomena in Ionized Gases, Prague, Czech Republic, July 15-20, 2007, Proc. on CD, 1594-1597

[30] Coudert J F, Rat V 2008 Influence of configuration and operating conditions on the electric arc instabilities of a plasma spray torch: role of acoustic resonance J. Phys. D: Appl. Phys.41, 205208

[31] Gonzales J J, Gleizes A, Freton P, Hlína J, Šlechta J 2002 Influence of gas flow rate on mixing of argon with air in thermal plasma jets Czech. J. Phys. 52, Suppl. D, D842-D849

\section{Figures:}

Figure 1. Experimental set-up.

Figure 2. Spectral characteristics of interference filter.

Figure 3. Optical radiation of argon in the spectral interval specified by the interference filter.

Figure 4. Frequency spectra at the axis and edge ( $3 \mathrm{~mm}$ from axis) of the plasma jet. Tangential gas

flow rate $23.5 \mathrm{slm}$, distance $4 \mathrm{~mm}$ from nozzle.

Figure 5. Maximal temperature as a function of time. Distance from nozzle $2 \mathrm{~mm}$, tangential flow rate $17 \mathrm{slm}$.

Figure 6. Maximal temperature as a function of time. Distance from nozzle $2 \mathrm{~mm}$, tangential flow rate $23.5 \mathrm{slm}$.

Figure 7. Evolution of temperature distributions $2 \mathrm{~mm}$ from nozzle in maximal current phase.

Tangential flow rate $17 \mathrm{slm}$, starting time 4,91 ms according to time coordinate in Fig. 4, interval between distributions $2.14 \mu$ s, interval between isotherms $200 \mathrm{~K}$. Size of depicted areas approx. 8x8 mm.

Figure 8. Evolution of temperature distributions $2 \mathrm{~mm}$ from nozzle in minimal current phase.

Tangential flow rate $17 \mathrm{slm}$, starting time $3.24 \mathrm{~ms}$ according to time coordinate in Fig. 4, interval between distributions $2.14 \mu$ s, interval between isotherms $200 \mathrm{~K}$. Size of depicted areas approx. 8x 8 $\mathrm{mm}$.

Figure 9. Average temperature distributions in $10 \mathrm{~ms}$ period. Tangential flow rate $17 \mathrm{slm}$, distance from nozzle $4 \mathrm{~mm}$.

Figure 10. Standard deviation of temperature distributions in $10 \mathrm{~ms}$ period. Tangential flow rate 17 slm, distance from nozzle $4 \mathrm{~mm}$. 


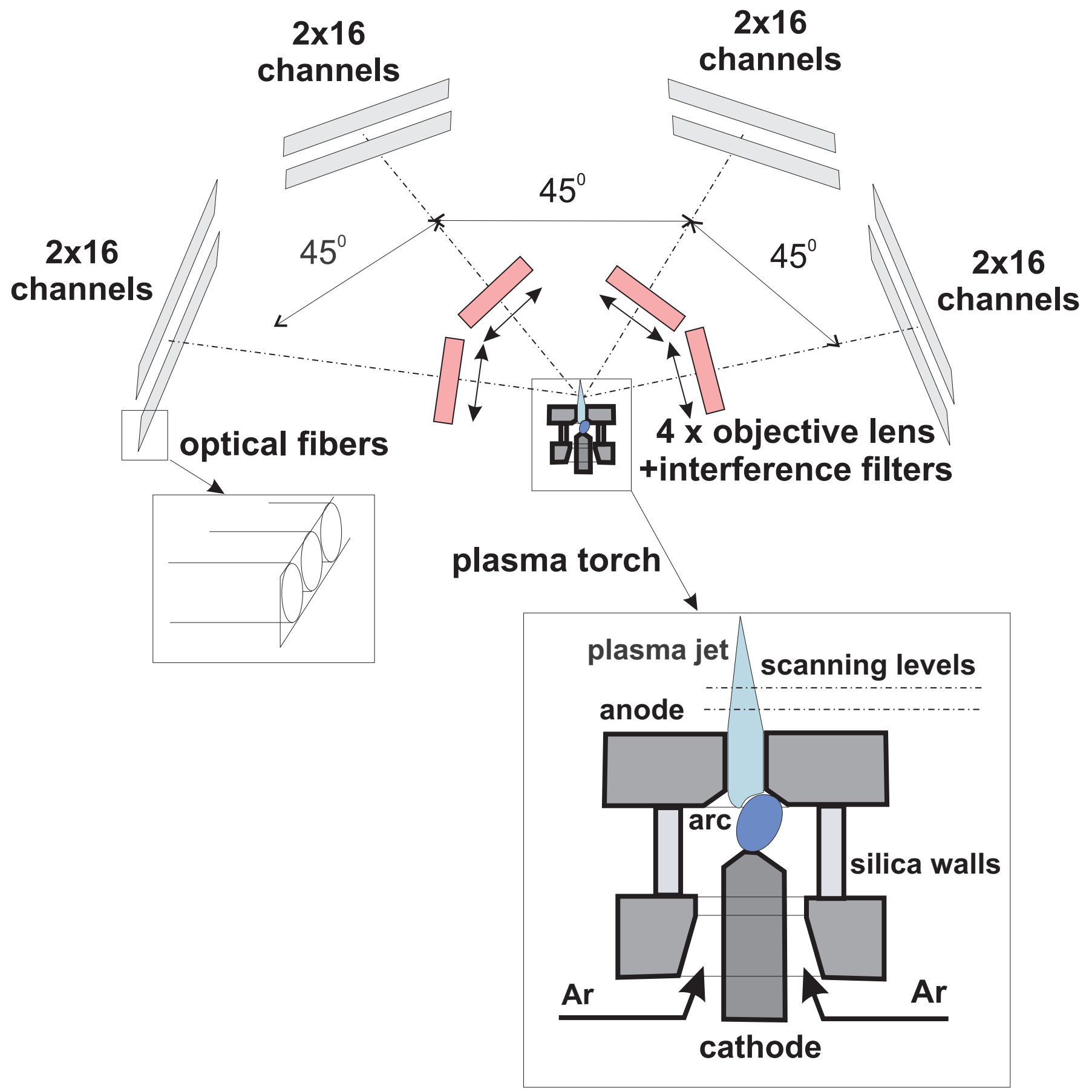

Figure 1 (f1.eps) 


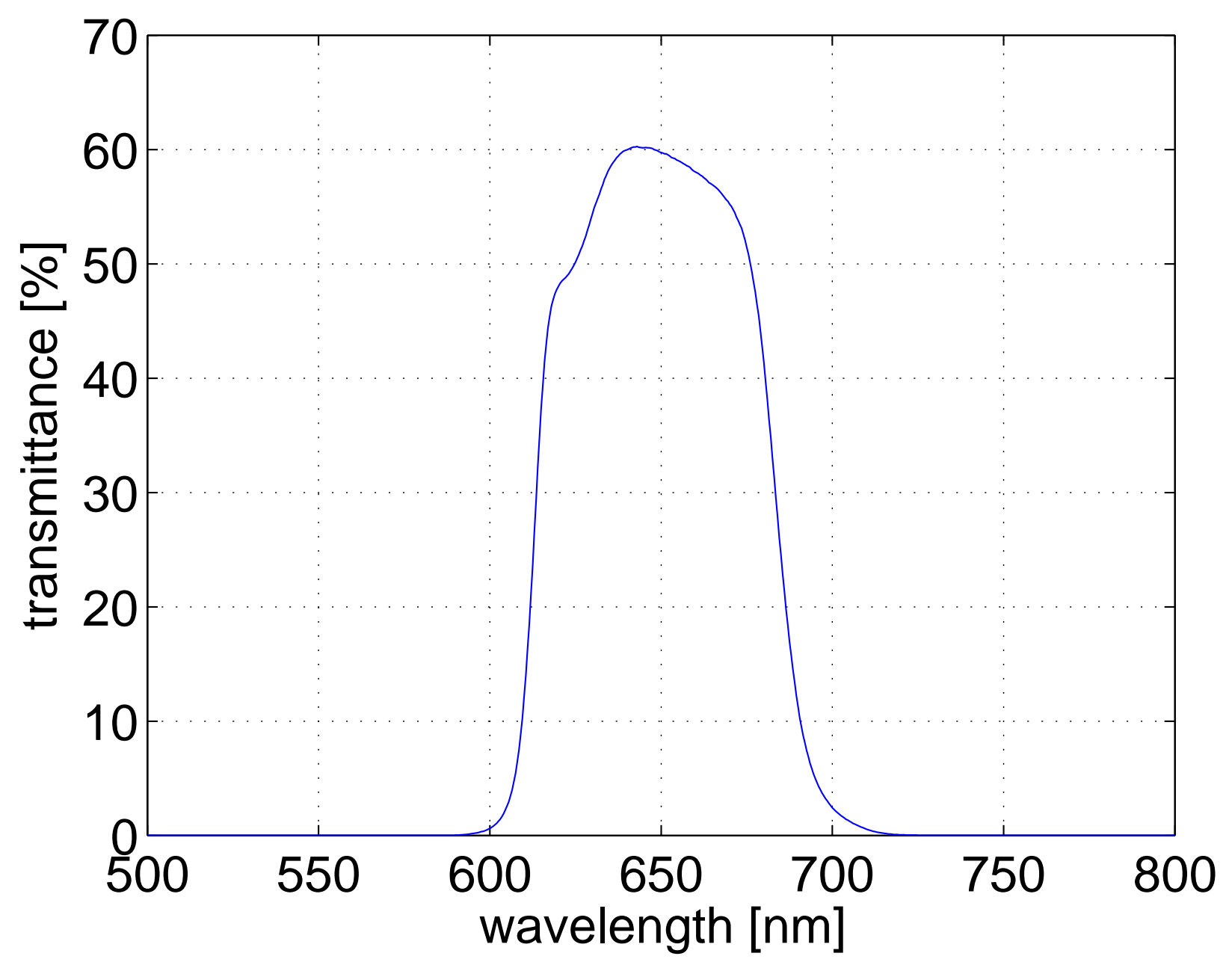

Figure 2 (f2.eps) 


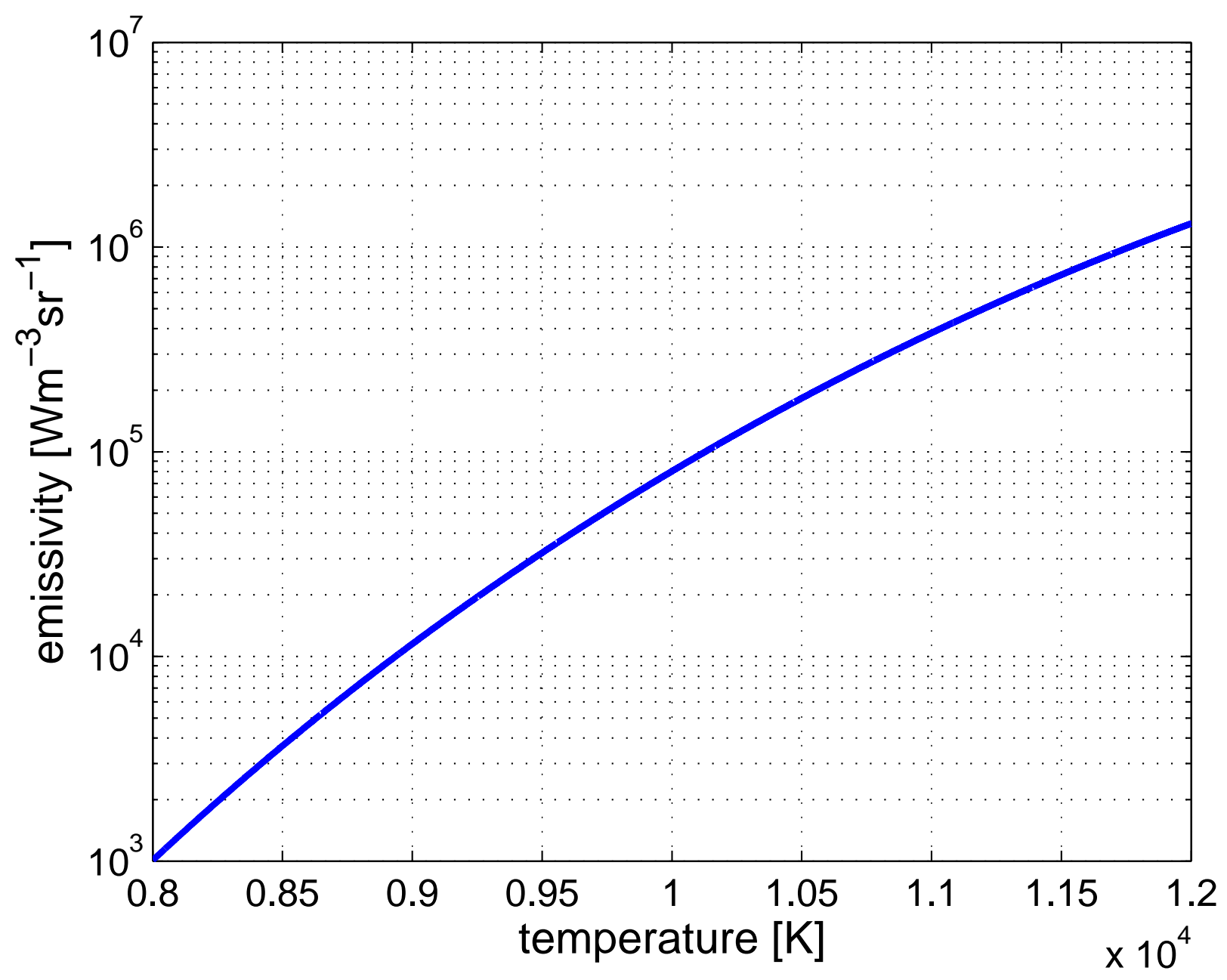

Figure 3 (f3.eps) 

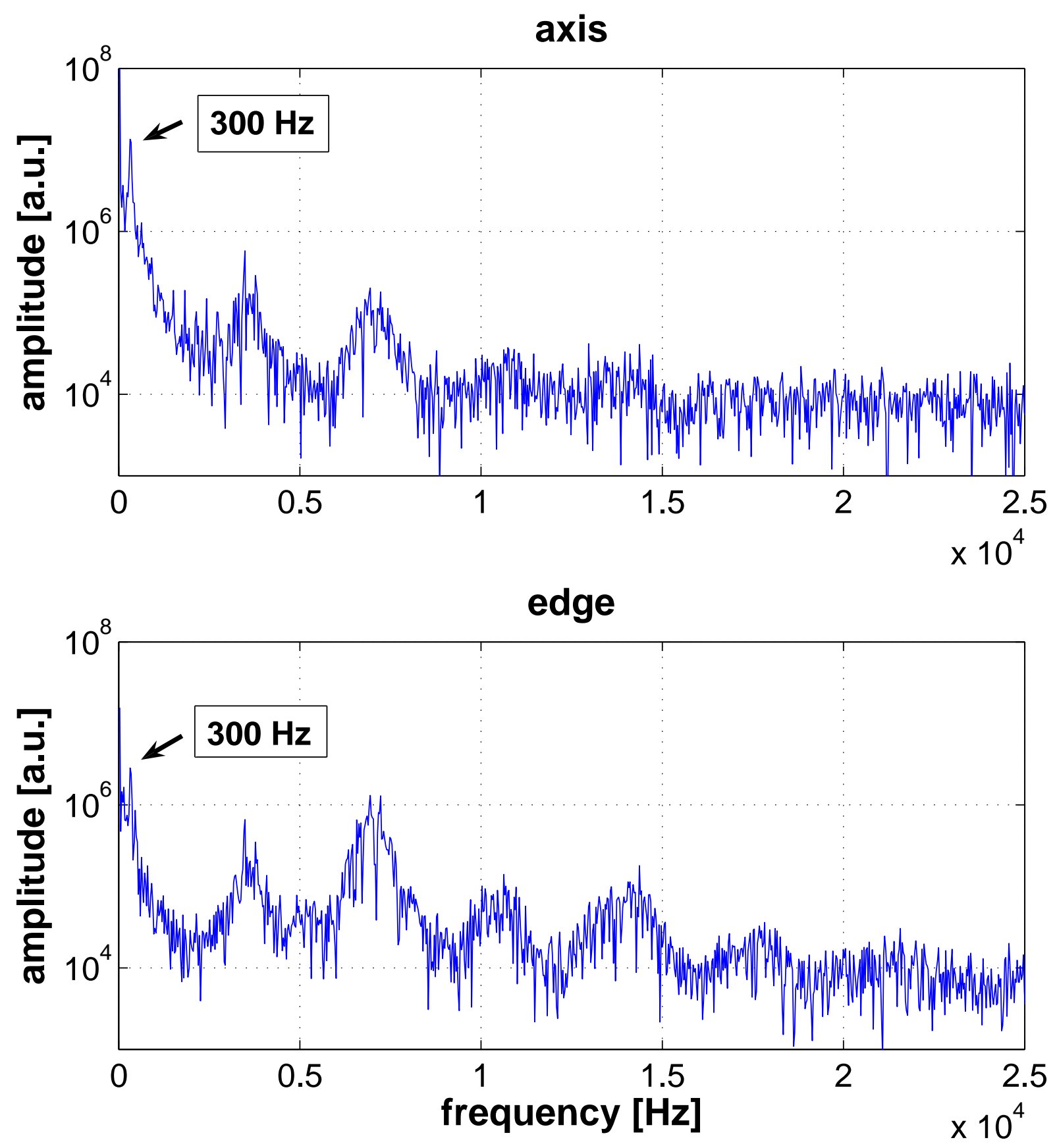

Figure 4 (f4.eps) 


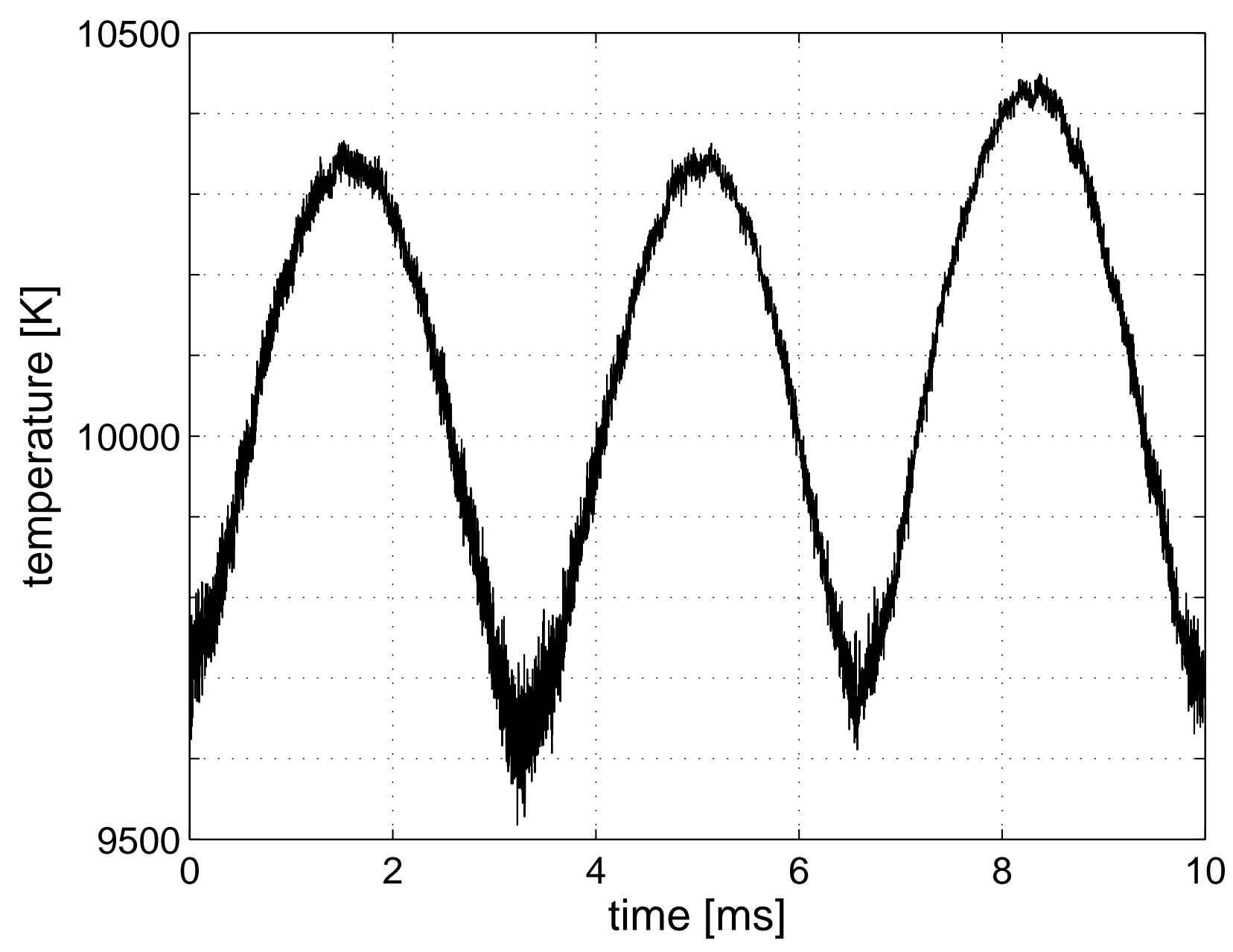

Figure 5 (f5.eps) 


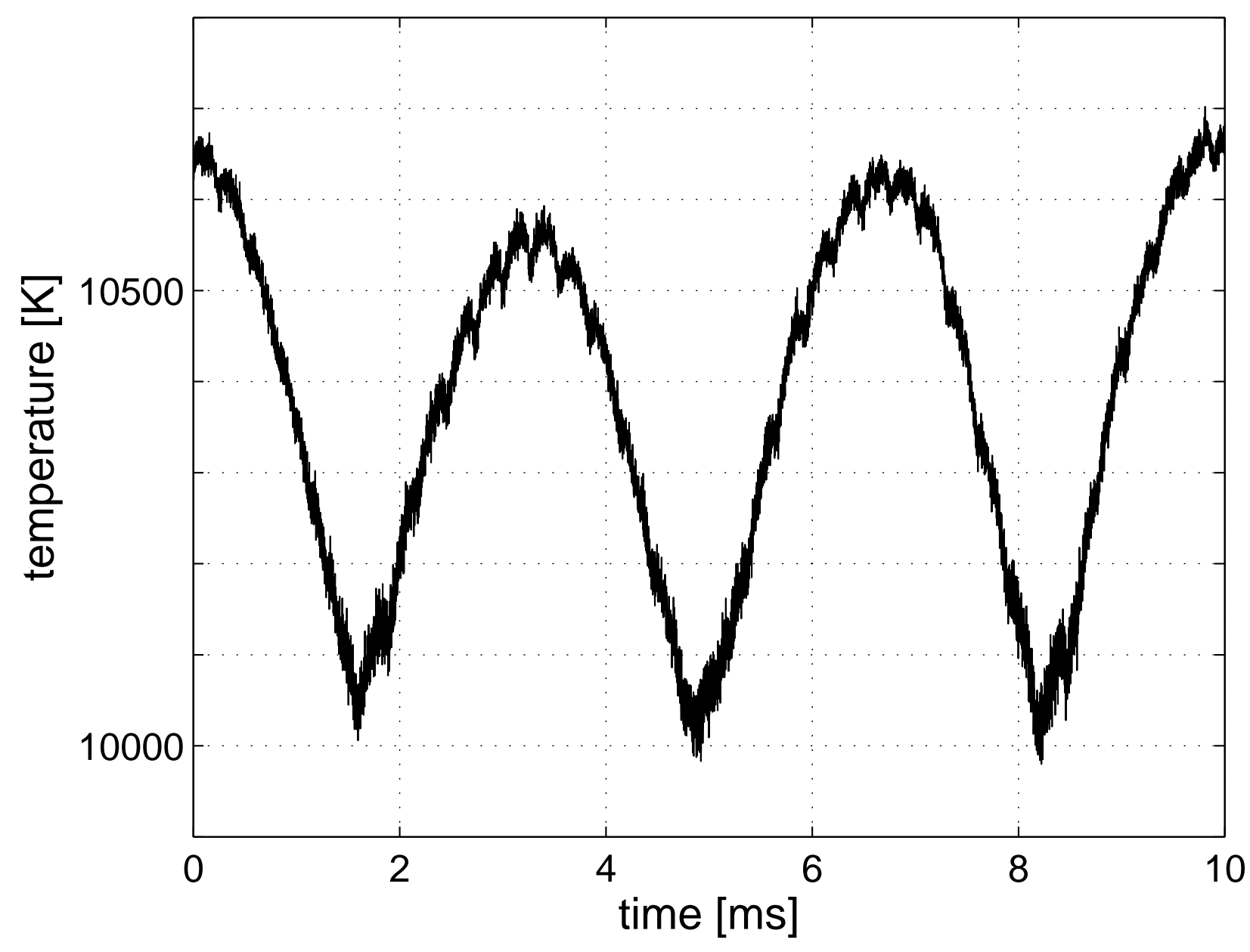

Figure 6 (f6.eps) 

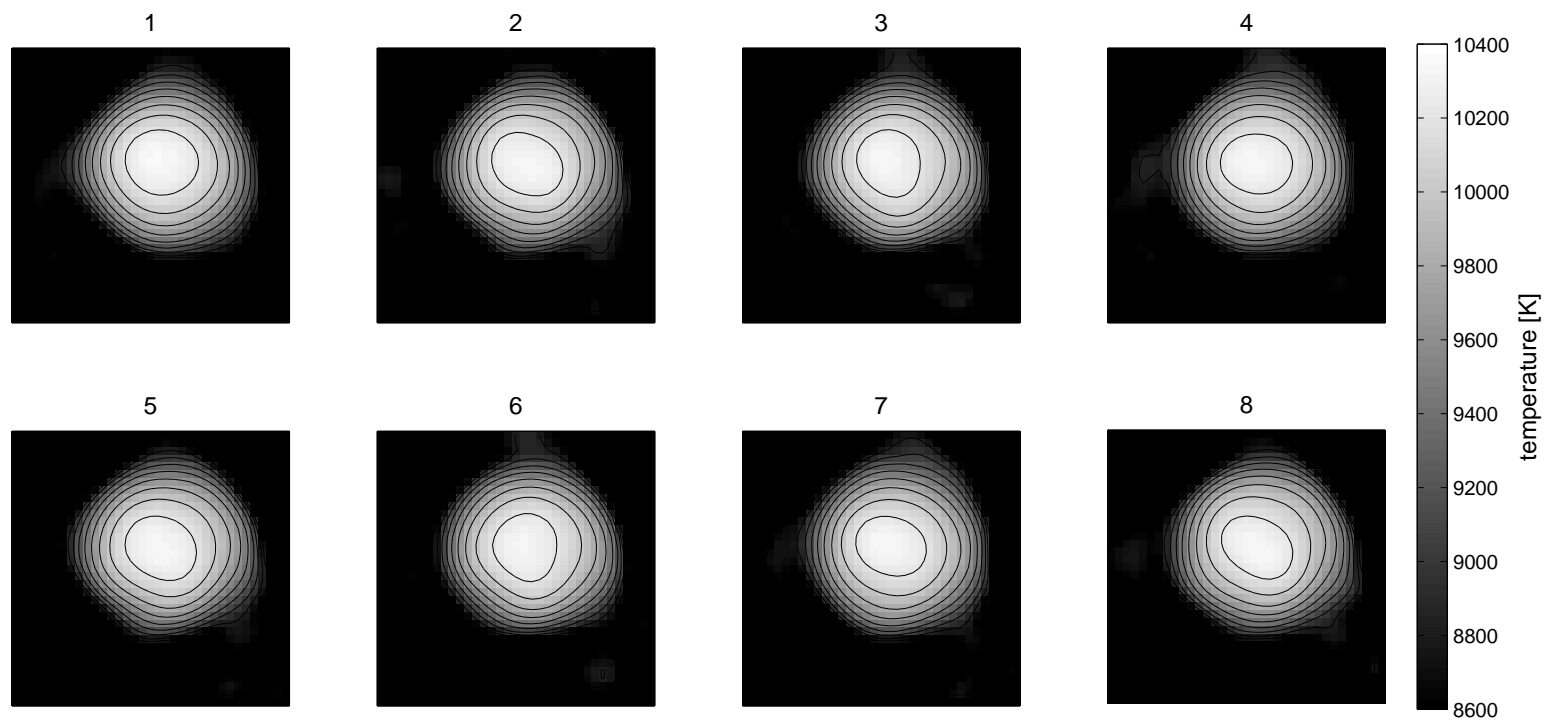

Figure 7 (f7.eps) 

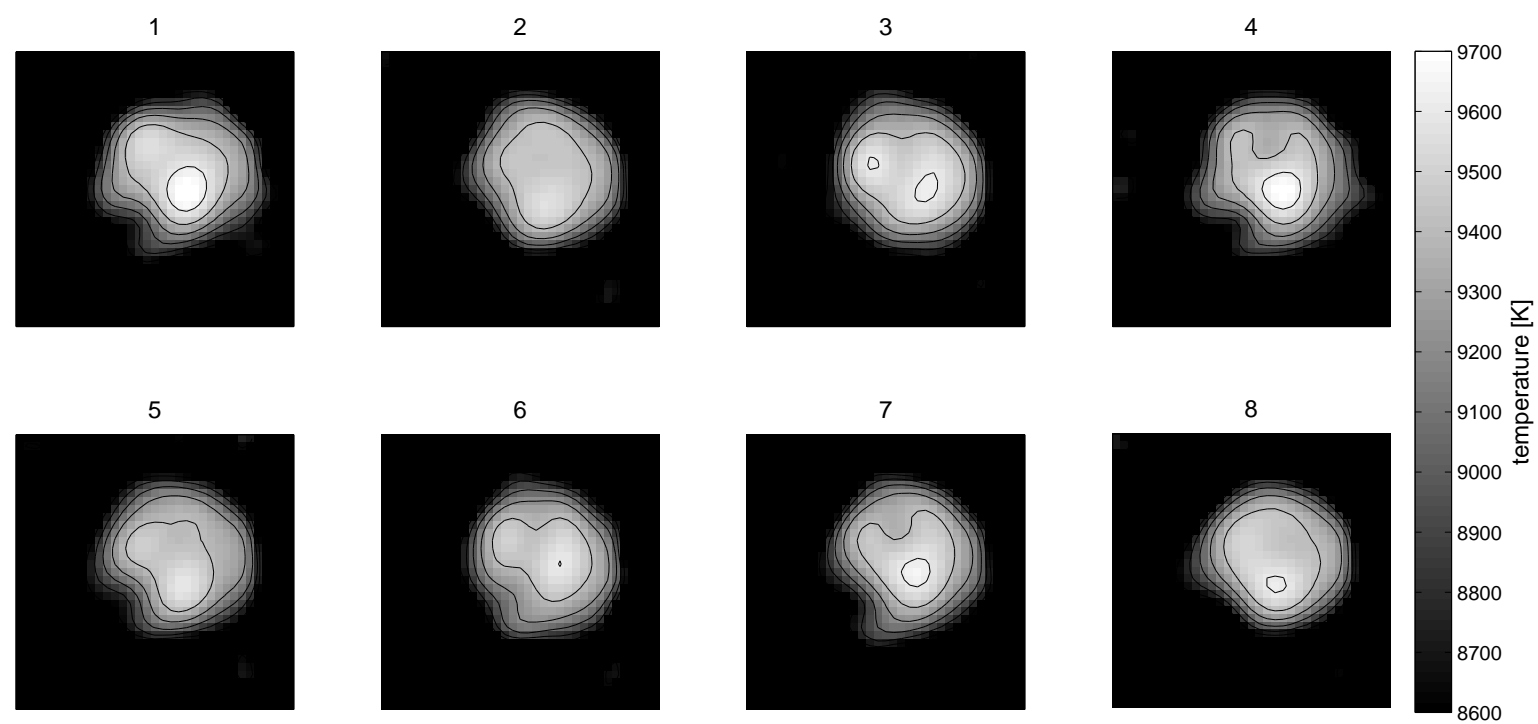

Figure 8 (f8.eps) 


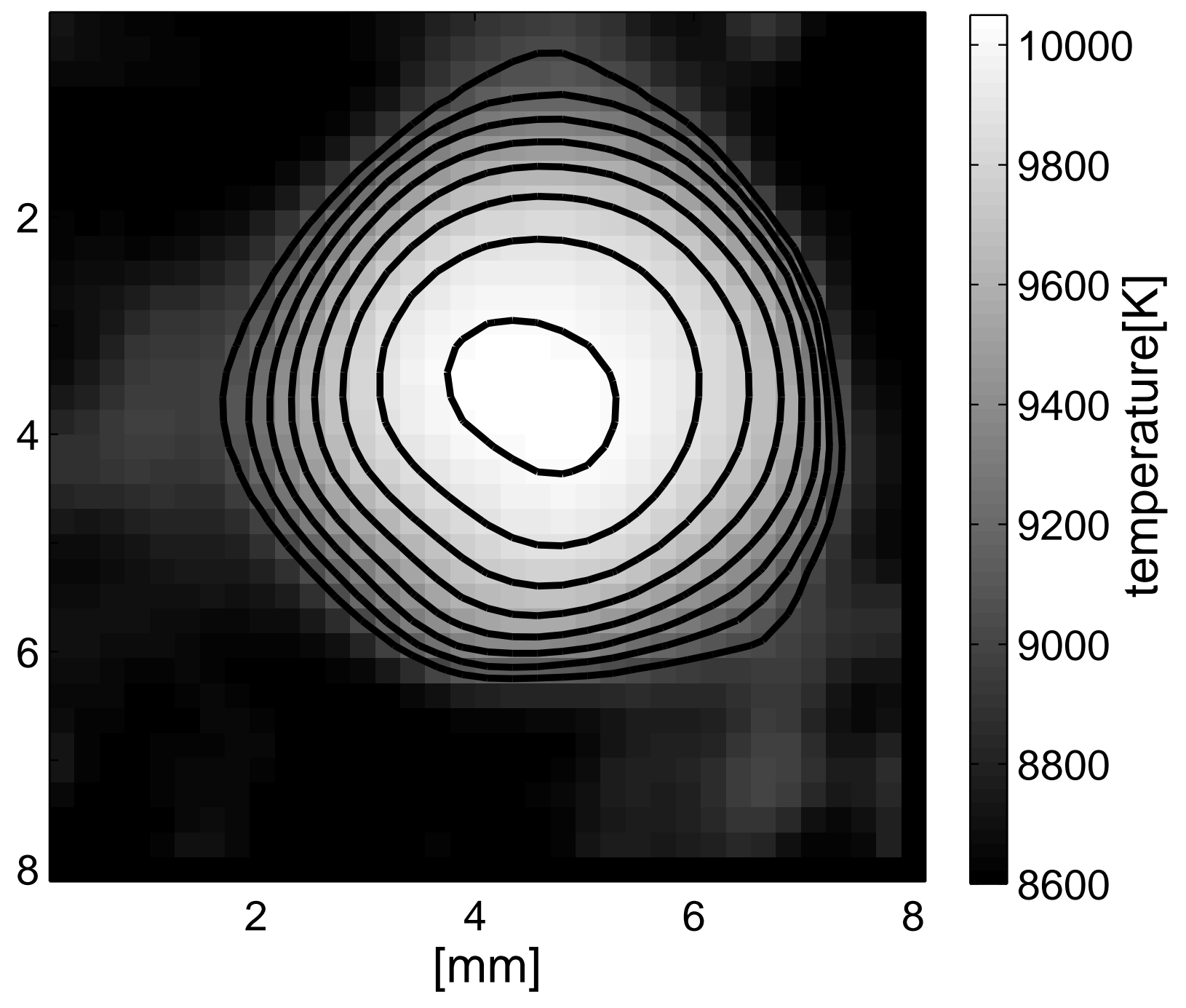

Figure 9 (f9.eps) 


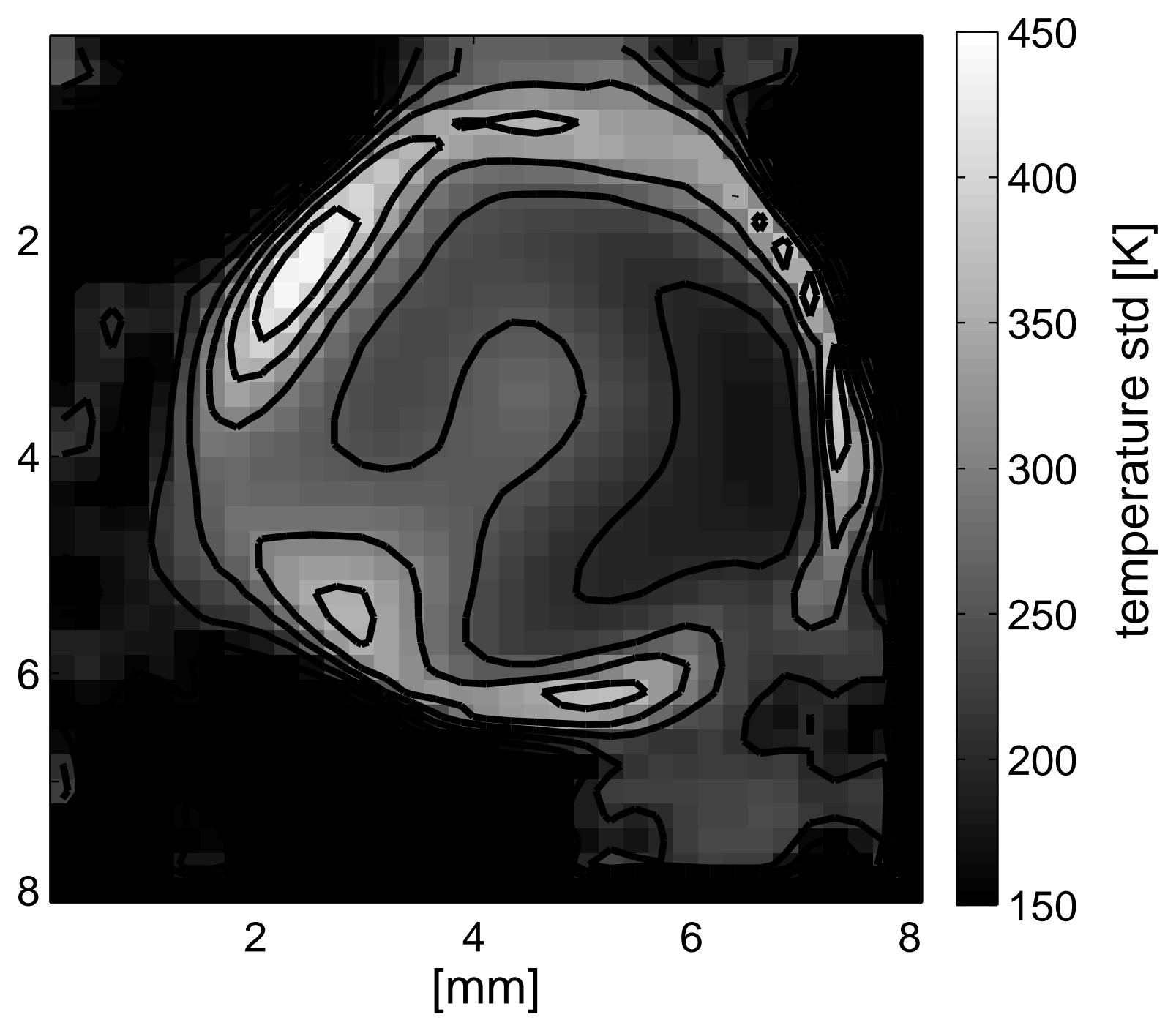

Figure 10 (f10.eps) 